\title{
Ajuga decumbens stimulates mesenchymal stem cell differentiation and regenerates cartilage in a rabbit osteoarthritis model
}

\author{
YOKO SAWADA $^{1}$, ATSUSHI SUGIMOTO ${ }^{1}$, TOMOHIRO OSAKI ${ }^{2}$ and YOSHIHARU OKAMOTO ${ }^{2}$ \\ ${ }^{1}$ Technology Research and Development Laboratory, Research and Development Headquarters, \\ Asahi Group Foods, Ltd., Moriya-shi, Ibaraki 302-0106; ${ }^{2}$ Department of Veterinary Clinical Medicine, \\ Faculty of Agriculture, Tottori University, Tottori 680-8553, Japan
}

Received January 24, 2017; Accepted September 13, 2017

DOI: $10.3892 / \mathrm{etm} .2018 .5981$

\begin{abstract}
In a previous study by our group, Ajuga decumbens extract (ADE) was demonstrated to decrease the number of osteoclasts in subchondral bone and to have a synergistic effect with glucosamine in improving cartilaginous injuries in a rabbit model of osteoarthritis. In the present study, a concentrate of the useful fraction of ADE, termed extra ADE (EADE), which includes higher concentrations of the active component 20-hydroxyecdysone, was evaluated for its efficacy to accelerate the healing of experimental cartilage injury. Cartilage injuries were surgically induced in rabbits by creating three holes; one in the articular cartilage of the medial trochlea and two in the trochlear sulcus of the distal femur. The rabbits were divided into the following four groups ( $\mathrm{n}=3$ in each): Control, $\operatorname{ADE}(0.5 \mathrm{~g} / \mathrm{kg})$, low dosage EADE $(0.05 \mathrm{~g} / \mathrm{kg}$; low EADE) and high dosage EADE $(0.5 \mathrm{~g} / \mathrm{kg}$; high EADE). ADE and EADE were dissolved in tap water and each dosage was orally administered every day for 3 weeks. At the end of the experimental period, histological analysis indicated that the cartilage matrix was regenerated in the low and high EADE groups. On counting of cells in the histological specimens, it was determined that the mean number of osteoclasts per 100 osteoblasts in subchondral bone was lower in the high EADE group compared with the control group. Furthermore, the results indicated that treatment with EADE $(1-100 \mu \mathrm{g} / \mathrm{ml})$ stimulated chondrogenic differentiation of mesenchymal stem cells and induced proteoglycan production to a greater extent than the control in vitro. EADE treatment (10 and $100 \mu \mathrm{g} / \mathrm{ml})$ was also observed to significantly attenuate interleukin-1 $\beta$-induced prostaglandin $\mathrm{E} 2$ production in chondrocytes $(\mathrm{P}<0.05)$. In summary, the results of the present study suggest that EADE
\end{abstract}

Correspondence to: $\mathrm{Dr}$ Tomohiro Osaki, Department of Veterinary Clinical Medicine, Faculty of Agriculture, Tottori University, 4-101 Koyama-Minami, Tottori 680-8553, Japan E-mail: tosaki@muses.tottori-u.ac.jp

Key words: Ajuga decumbens, anti-inflammation, cartilage regeneration, mesenchymal stem cell, osteoarthritis, osteogenesis may have greater curative effects on bone injury compared with the currently used therapeutic ADE.

\section{Introduction}

Osteoarthritis (OA) is a chronic degenerative joint disorder that causes pain, stiffness, and limitation in the range of joint motion (1). OA is a major public health problem in the elderly population; OA of the knee, as one of the most common forms of OA, affected up to 25 million people in Japan in 2006 (1). OA is caused by articular cartilage degeneration, which involves fibrillation of the articular surface and a decrease in the size and aggregation of proteoglycan (PG) (2-4). These alterations are principally the result of aging-associated changes in chondrocyte function, including a decrease in synthetic activity, that decrease their ability to maintain the tissue (4). The dietary supplement D-glucosamine hydrochloride $(\mathrm{GlcN})$ has previously been used as a safe and effective treatment for the management of OA symptoms (5). GlcN has been documented to decrease chemical mediators in chondrocytes (6), induce cartilaginous matrix regeneration in experimental OA and restore the articular surface at the injury site by affecting PG and collagen production $(7,8)$. However, previous results suggest that GlcN alone or in combination with other supplements does not effectively reduce pain in patients with knee OA (9), though it has been reported that a combination of GlcN and chondroitin sulfate was effective when used as a treatment in a subgroup of patients with moderate-to-severe knee pain (9). To develop a more effective supplement for the treatment of OA, the aim of the present study was to investigate a compound that may have therapeutic effects in this disease.

Ajuga decumbens (AD), a naturally occurring herb that has a history of use as a pain relief medicine in Japan (10), was selected as the candidate supplement for the treatment of OA. $\mathrm{AD}$ extract (ADE) has previously been reported to have preventative effects against osteoporosis (10), to decrease the number of osteoclasts following subchondral bone damage, and to work synergistically with GlcN to improve cartilaginous injury in a rabbit OA model (11). 20-hydroxyecdysone, an active component of ADE, has also been demonstrated to decrease the number of osteoclasts following subchondral bone damage in cartilaginous injuries (11). Furthermore, 20-hydroxyecdysone has beneficial effects on epiphyseal cartilage tissue and 
trabecular bone in ovariectomized rats (12), and ADE has been reported to induce subchondral bone regeneration (11). However, the mechanism underlying ADE-induced regeneration in the cartilage matrix is unclear. Chondrocytes have been implicated as a critical component that influence repair during cartilage degradation (13). Chondrocytes are present in hyaline cartilage and develop from the highly regulated differentiation of mesenchymal stem cells (MSCs), mesodermal-derived stem cells present in a number of fetal and adult tissues (13). MSCs have recently been applied as a treatment for OA in clinical trials due to their regeneration potential and anti-inflammatory effects, and therapeutic effects of chondrogenic-differentiated MSCs on OA were observed (13). Although transplanted native MSCs may be problematic due to their multipotent differentiation activity, the use of pre-differentiated MSCs may increase the speed of defect healing (14). Furthermore, some compounds, such as kartogenin have been reported to affect MSC differentiation and exert therapeutic effects on joint injury (15).

In the present study, a concentrate of the effective fraction of ADE, termed extra ADE (EADE), was evaluated for its therapeutic effect in a rabbit model of cartilage injury. In addition, the potential effects of EADE on MSC-differentiation and anti-inflammatory responses in chondrocytes were assessed to elucidate the molecular mechanisms underlying cartilage regeneration following injury.

\section{Materials and methods}

Preparation of $A D E$ and $E A D E$. The whole dried AD plant was refluxed with aqueous ethanol and $A D$ was extracted to produce ADE. EADE was obtained from the extract using semi-polarity resins and the concentrated fraction had $>1 \mathrm{wt} \%$ 20-hydroxyecdysone. ADE and EADE were purchased ready made from Matsuura Yakugyo Co., Ltd. (Aichi, Japan).

Animal model. The animal model was established using a previously published method $(11,16)$. A total of 18 healthy Japanese Albino female rabbits, 12 weeks old and weighing $2.0 \pm 0.5 \mathrm{~kg}$ were purchased from Shimizu Laboratory Supplies Co., Ltd. (Kyoto, Japan) and acclimated for 1 week in the laboratory environment. The animals were housed at $25^{\circ} \mathrm{C}$ in $50-60 \%$ relative humidity, in a $12 \mathrm{~h}$ light/dark cycle, with free access to RC4 food (Oriental Yeast Co., Ltd., Tokyo, Japan) and tap of water. The use of the animals and the procedures followed were approved by the Animal Research Committee of Tottori University (Tottori, Japan).

Experimental procedures. The analgesic xylazine hydrochloride (Selactar ${ }^{\circledR}$; Bayer Yakuhin, Ltd., Osaka, Japan), was administered $(10 \mathrm{mg} / \mathrm{kg})$ as premedication. Following sedation, induction of anesthesia was performed in an anesthetizing box with a mixture of 5\% isoflurane (Intervet; Merck KGaA, Darmstadt, Germany) in oxygen. Anesthesia was maintained by inhalation of a mixture of $3 \%$ isoflurane in oxygen using a mask. The fur at the left knee joint was clipped and the area was disinfected with chlorhexidine solution (Hibiscrub; Sumitomo Dainippon Pharma Co., Ltd., Osaka, Japan) and 70\% alcohol. Approaching from the lateral portion of the knee joint, an incision was made vertically from the central part of the femur toward the tibial tuberosity. The articular capsule was incised, and the patella of the stifle joint was exposed completely by artificially dislocating the patella toward the medial side. Three holes measuring $2 \mathrm{~mm}$ in diameter and $4 \mathrm{~mm}$ in depth were made using a hand drill (Micro-engine BL-F; Osada Electric Co., Ltd., Tokyo, Japan) at the articular cartilage of the medial trochlea (one hole) and the trochlear sulcus (two holes) of the distal femur. The wound was rinsed with saline solution and the articular capsule was sutured and closed with a synthetic absorbent thread (3-0 PDSII; Johnson \& Johnson, New Brunswick, NJ, USA). The subcutaneous tissues and skin were sutured with nylon (USP 3-0 suture; Suprylon, Vomel, Germany). During the 1-week period following surgery, the wound surface was disinfected with povidone-iodine once daily, and $10 \mathrm{mg} / \mathrm{kg}$ oxytetracycline (Pfizer, New York, NY, USA) was subcutaneously administered twice daily to prevent infection.

$A D E$ and EADE administration. Rabbits were divided into four groups as follows: Control, ADE, low dosage EADE (low EADE) and high dosage EADE (high EADE) $(n=3$ in each). ADE contained 0.04\% 20-hydroxyecdysone and EADE contained $1.38 \%$ 20-hydroxyecdysone as specified by the suppliers. Rabbits were administered with the following: ADE group, $500 \mathrm{mg} \mathrm{ADE} / \mathrm{kg} /$ day $(0.2 \mathrm{mg} / \mathrm{kg} /$ day 20-hydroxyecdysone); low EADE group, $50 \mathrm{mg}$ EADE/ $\mathrm{kg} /$ day $(0.69 \mathrm{mg} / \mathrm{kg} /$ day 20-hydroxyecdysone); high EADE group, $500 \mathrm{mg}$ EADE/kg/day (6.9 mg/kg/day 20-hydroxyecdysone). ADE and EADE were dissolved in tap water and each dosage was orally administered every day for 3 weeks. The control group had free access to tap water. At 3 weeks post-surgery, the rabbits were euthanized by overdose (160 mg/kg; intravenous injection) of pentobarbital (Sumitomo Dainippon Pharm Co., Ltd., Osaka, Japan). The stifle joints were opened and observed macro- and microscopically to assess the injured cartilage.

Assessment of macroscopic changes. For macroscopic analysis, the extent of restoration within the surgical holes was scored as previously reported (17). The restoration scoring was as follows: $<50 \%$ restored, 0 points; $>50-50 \%, 1$ point; $>60-80 \%$ restored, 2 points; $>80 \%$ restored, 3 points. The degree of restoration was scored separately for the trochlear sulcus and the medial trochlear ridge in each rabbit to calculate a group mean for each region. The mean representative of both areas was then calculated. The scoring was performed by a veterinarian.

Assessment of histological changes. Histological assessment was performed on the femurs of the rabbits in each group. The recovered left femur was fixed in $10 \%$ neutral buffered formaldehyde solution for $1 \mathrm{~h}$ at room temperature. Following fixation, the stifle joint that had been operated on was trimmed to a thickness of $5 \mathrm{~mm}$ and decalcified by agitating in $5 \%$ formic acid solution at $25^{\circ} \mathrm{C}$ for 1 day. The tissue was subsequently soaked in $5 \%$ sodium sulfate solution at $25^{\circ} \mathrm{C}$ for 1 day to neutralize and was subsequently washed at $25^{\circ} \mathrm{C}$ for $\sim 10 \mathrm{~h}$ under running water. The tissue was then embedded in paraffin and cut into 5- $\mu \mathrm{m}$ slices using a microtome. Staining was performed using hematoxylin and eosin (H\&E), Safranin $\mathrm{O}$ and Alcian blue methods. All methods were performed at $25^{\circ} \mathrm{C}$ for $10 \mathrm{~min}$. Images of restored areas, articular cartilage 
and the growth zone were captured using an OpticLab H850 (Plustek, Tokyo, Japan) and evaluated with ImageJ software version 1.49 (National Institutes of Health, Bethesda, MD, USA). The depth of restoration in the cartilaginous and subchondral bone matrices were measured based on the H\&E staining according to a previously published method $(11,16)$. With Safranin O staining, the red pixels indicating the presence of PGs were counted, while non-specific colored pixels were not included. With Alcian blue staining, the indigo pixels indicating the presence of glycosaminoglycans (GAGs) were also counted. The difference between the restored substances at the injured sites in all groups was recorded based on observation with a light microscope (BX51-FL; Olympus Corporation, Tokyo, Japan). The proportion of the pixels counted in the desired color from a total of 120,000 pixels (random sampling of 20,000 pixels at six locations in each cartilaginous matrix) was then calculated the number of pixels using an image processing technique in ImageJ. The number of osteoclasts (multinucleated) and osteoblasts (mononuclear) in the subchondral bone were recorded in 10 random areas under a light microscope, and the mean number of osteoclasts per 100 osteoblasts was calculated.

Culture and cytochemical staining of human MSCs (hMSCs). hMSCs derived from umbilical cord matrix (hMSC-UC cell line) were obtained from PromoCell GmbH (Heidelberg, Germany). The cells were seeded at $1 \times 10^{5}$ cells/well in a 96-well plate and cultured at $37^{\circ} \mathrm{C}$ in a humidified atmosphere containing $5 \% \mathrm{CO}_{2}$ for 3 weeks in different media. Negative control cells were cultured in $0.2 \mathrm{ml}$ mesenchymal stem cell growth medium which was purchased from PromoCell GmbH. Positive control cells were cultured in a complete chondrogenic differentiation medium (PromoCell $\mathrm{GmbH}$ ) and EADE cells were cultured in different concentrations of EADE $(1,10$ or $100 \mu \mathrm{g} / \mathrm{ml}$ ) resolved in chondrogenic differentiation medium. The media were changed twice weekly. Following the culture period, histochemical analysis of chondrogenic differentiation was assessed by PG accumulation, as measured by staining of cell clusters with Alcian blue. Cells were first rinsed with PBS three times and fixed with $100 \%$ methanol for $10 \mathrm{~min}$ at room temperature. Staining was accomplished by applying a solution of $0.1 \%$ Alcian blue pH 2.5 (Nacalai Tesque, Kyoto, Japan) to the cells for $18 \mathrm{~h}$ at $4^{\circ} \mathrm{C}$. To quantify the intensity of the staining, the stained culture plates were rinsed with $0.1 \mathrm{~N} \mathrm{HCl}$ twice, and each well was extracted with $6 \mathrm{M}$ guanidine- $\mathrm{HCl}$ overnight at room temperature. The optical densities of the cell spheroids and extracted dye were measured at $630 \mathrm{~nm}$ with a microplate reader.

Chondrocyte culture and measurement of prostaglandin E2 $\left(P G E_{2}\right)$. Human chondrocytes (HC) derived from normal human femoral cartilage were obtained from Cell Applications, Inc. (Merck KGaA). The cells were maintained in chondrocyte growth medium which was purchased from Cell Applications, Inc. (Merck $\mathrm{KGaA}$ ) at $37^{\circ} \mathrm{C}$ in a humidified atmosphere containing $5 \% \mathrm{CO}_{2}$. For treatment with interleukin (IL)-1 $\beta$ (PeproTech, Inc., Rocky Hill, NJ, USA), the cells were seeded at $1 \times 10^{4}$ cells/well in a 24 -well plate. Following overnight incubation at $37^{\circ} \mathrm{C}$ in a humidified atmosphere containing $5 \% \mathrm{CO}_{2}$, the growth medium was changed and cells were stimulated with $1 \mathrm{ng} / \mathrm{ml} \mathrm{IL}-1 \beta$ in the presence of 10 or $100 \mu \mathrm{g} / \mathrm{ml}$ of EADE prior to further incubation at $37^{\circ} \mathrm{C}$ in a humidified atmosphere containing $5 \% \mathrm{CO}_{2}$ for $24 \mathrm{~h}$. Supernatants were subsequently collected to measure the levels of $\mathrm{PGE}_{2}$ using a $\mathrm{PGE}_{2}$ Parameter Assay kit (R\&D Systems, Inc., Minneapolis, MN, USA) according to the manufacturer's protocol.

Statistical analysis. Data are expressed as the mean + standard deviation of the mean. Two groups of data were analyzed using a Student's t-test. Multiple groups of data were analyzed using one way analysis of variance followed by a Dunnett's post hoc test. $\mathrm{P}<0.05$ was considered to indicate a statistically significant difference. Microsoft Excel 2007 (Microsoft Corporation, Redmond, WA, USA) was used for statistical analyses using t-tests, and IBM SPSS version 19.0 (IBM Corp., Armonk, NY, USA) was used for the other tests.

\section{Results}

\section{Effect of EADE in vivo}

Macroscopic effects of EADE on cartilage regeneration. In the control group, there was only a slight degree of restoration at the trochlear sulcus and medial trochlear ridge (Fig. 1). In the ADE and low and high EADE groups, the degree of restoration was markedly increased compared with the control group (Fig. 1). Notably, the degree of restoration in the trochlear sulcus was significantly greater in the high EADE group compared with the control group $(\mathrm{P}<0.01$; Fig. 1A); however, the degree of restoration in the medial trochlear ridge did not differ significantly between the groups (Fig. 1B). Meanwhile, the mean restoration score, obtained by pooling the data from the trochlear sulcus and medial trochlear ridge, was significantly increased in the high EADE group compared with the control group $(\mathrm{P}<0.01$; Fig. $1 \mathrm{C})$.

Effect of EADE on cartilage regeneration assessed by histological H\&E staining. Representative H\&E staining images of the trochlear sulcus at 3 weeks post-surgery are presented in Fig. 2. Tissue injury remained visible in the control group (Fig. 2A). In contrast, tissue regeneration was apparent in the cartilage and subchondral matrices of the ADE, low EADE and high EADE groups, with the defective areas filled with proliferating fibroblasts, cartilaginous cells and subchondral bone matrix, and the surface of the wound area covered with regenerated connective tissue (Fig. 2B-D). At a deeper level, the bone trabecular was regenerated and filled with proliferating cells (fibroblasts, cartilaginous cells and fibrous cartilage), giving it an appearance similar to that of mature cartilaginous substrates in the ADE, low EADE and high EADE groups. In the deeper zone, the subchondral bone matrix was markedly regenerated in the ADE, low EADE and high EADE groups (Fig. 2B-D). The cancellous bone structure was also partially regenerated in these groups, particularly in the high EADE group (Fig. 2D). Fig. 3 depicts the proportions of restoration in the cartilage matrix (Fig. 3A-C) and subchondral bone matrix (Fig. 3D-F). The proportion of regenerated cartilage matrix in the trochlear sulcus was greater in the ADE, low EADE and high EADE groups compared with the control group (Fig. 3A). At the medial trochlear ridge, the degree of restoration was 

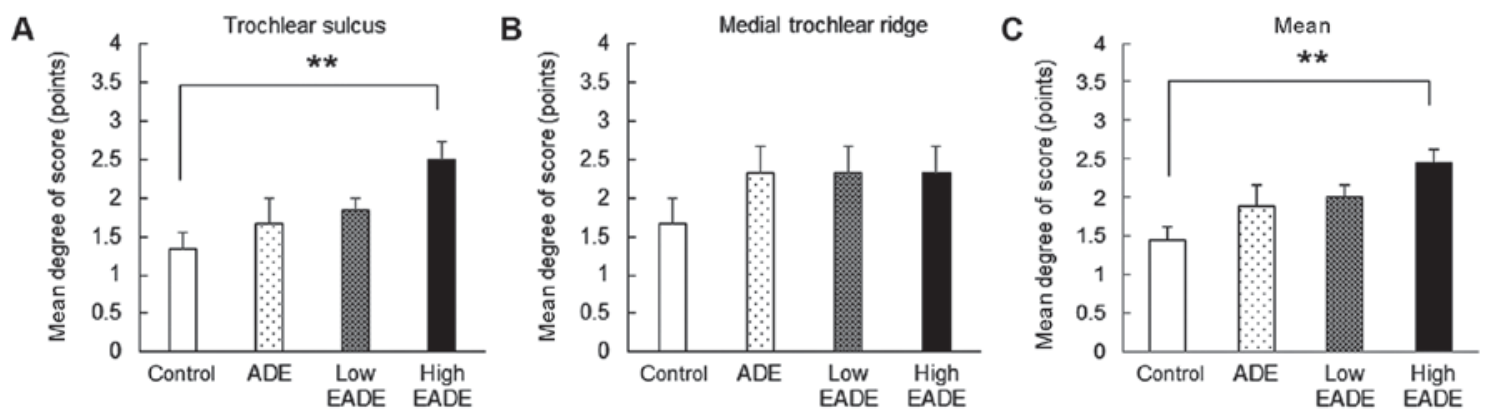

Figure 1. Restoration of the cartilage holes. The degree of restoration in the surgical holes was quantified using the following scoring system: $<50 \%, 0$ points; $>50-60 \%, 1$ point; $>60-80 \%, 2$ points; $>80 \%, 3$ points. The graphs present the scores for the (A) two holes in the trochlear sulcus, (B) single hole in the medial trochlear ridge and $(\mathrm{C})$ mean of the combined data for all holes. ${ }^{* *} \mathrm{P}<0.01$. ADE, Ajuga decumbens extract; EADA, extra ADE.

A

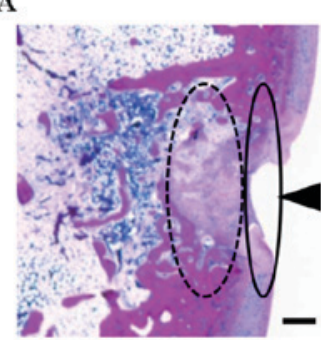

B

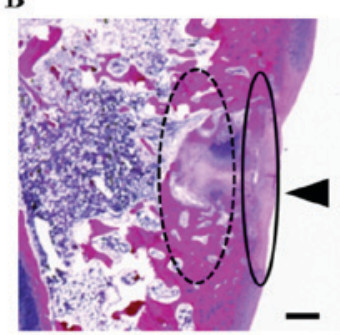

C

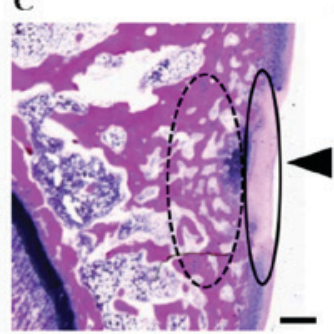

D

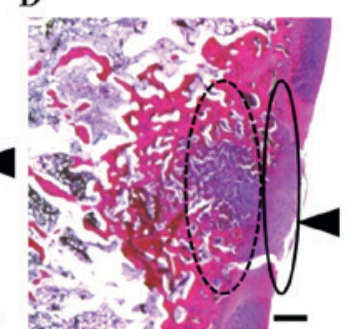

Figure 2. Hematoxylin and eosin staining of the trochlear sulcus at 3 weeks post-surgery. (A) Tissue damage (black arrowheads) remained visible in the control group. The surgical holes were filled with fibrous cartilage, like proliferating fibroblasts, cartilaginous cells (solid circle) and subchondral bone matrix (broken circle) in the (B) ADE and (C) low EADE groups. (D) In the high EADE group, all holes were fully filled with cartilaginous matrix (solid circle) and subchondral matrix (broken circle). Scale bar, $500 \mu \mathrm{m}$. ADE, Ajuga decumbens extract; EADA, extra ADE.

markedly greater in the high EADE group compared with the control group (Fig. 3B), and on pooling of the data, the high EADE group exhibited a significantly greater combined mean depth of the two areas compared with the control group $(\mathrm{P}<0.05$; Fig. 3C). The proportion of regenerated subchondral bone matrix in the trochlear sulcus and the medial trochlear ridge, as well as the mean combined depth, was greater in the ADE, low EADE and high EADE groups compared with the control group (Fig. 3D-F); however, no significant differences were observed.

Effect of EADE on cartilage regeneration assessed by histological Safranin $O$ and Alcian blue staining. To evaluate cartilaginous matrix regeneration, histological staining with Safranin O and Alcian blue was performed. Representative Safranin $O$ and Alcian blue staining images of the trochlear sulcus are presented in Fig. 4. On Safrinin O staining, PGs in the cartilage matrix were stained dark red; a small degree of staining of the cartilage matrix was observed in the control group, whereas the cartilage matrix was stained more strongly in the ADE, low EADE and high EADE groups as determined by image analysis (Fig. 4A). In tissues stained with Alcian blue, the GAGs in the cartilage matrix were stained blue. The stained areas and color strength were similar to what was observed with Safranin O staining; stronger staining was observed in the cartilage matrix of the trochlear sulcus in the ADE, low EADE and high EADE groups compared with the control group (Fig. 4B). The image analysis of the cartilage matrix following Safranin $\mathrm{O}$ and Alcian blue staining is presented in Fig. 5. In the trochlear sulcus, the regenerated areas were significantly increased in the low and high EADE groups $(\mathrm{P}<0.05)$, with the two staining methods resulting in similar results (Fig. 5A and D). In the medial trochlear ridge, the strongest staining was observed in the high EADE group (Fig. 5B and E). Meanwhile, the mean of the total stained area was significantly higher in the low and high EADE groups compared with the control group $(\mathrm{P}<0.05$; Fig. 5C and $\mathrm{F})$, and collectively, the results suggested that EADE increased the number of GAGs and PGs in the cartilage matrix in a dose-dependent manner.

Effect of EADE on osteogenesis assessed by histological $H \& E$ staining. To evaluate the effect of EADE on the balance of osteogenesis, osteoclasts and osteoblasts were counted in the subchondral bone following histological H\&E staining (Fig. 6). In the control group, a marked number of osteoclasts were present, while few osteoblasts were observed (Fig. 6A). By contrast, in the ADE group, fewer osteoclasts were observed (Fig. 6B), and in the low and high EADE groups, the numbers of osteoblasts were increased (Fig. 6C and D). The mean number of osteoclasts was significantly reduced in the high EADE group at the trochlear sulcus compared with the control group (Fig. 7A; $\mathrm{P}<0.05$ ); however, the mean number of osteoclasts at the medial trochlear ridge did not differ significantly between the groups (Fig. 7B). Similarly, across the trochlear sulcus and medial trochlear ridge regions, the numbers of osteoclasts did not differ significantly (Fig. 7C). Subsequently, the mean number of osteoclasts per 100 osteoblasts in each group was calculated. No significant differences in the number of osteoclasts per 100 osteoblasts 
A
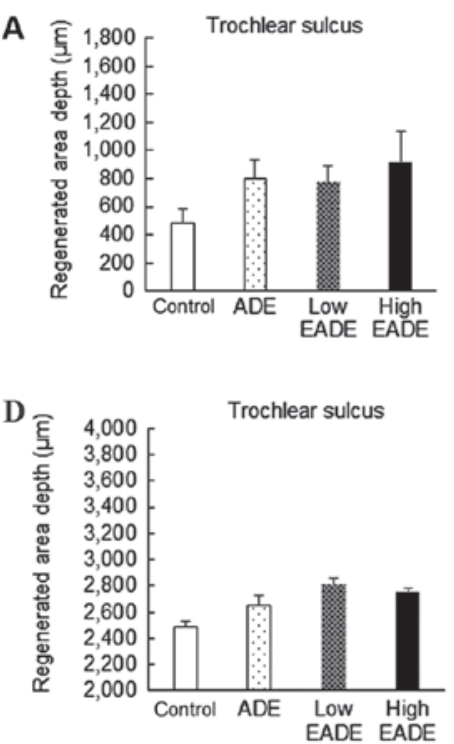

B

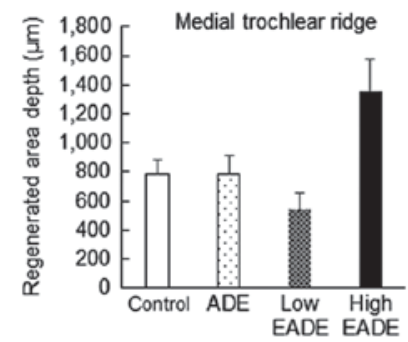

E

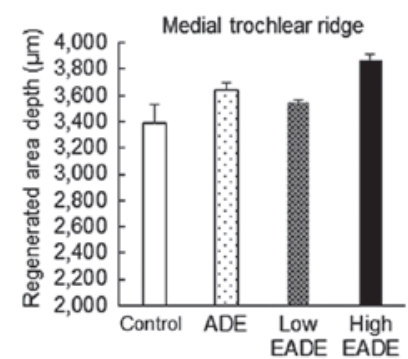

C

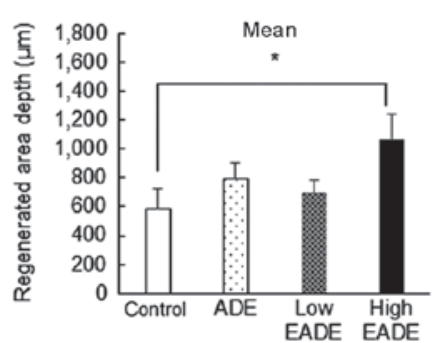

$\mathbf{F}$

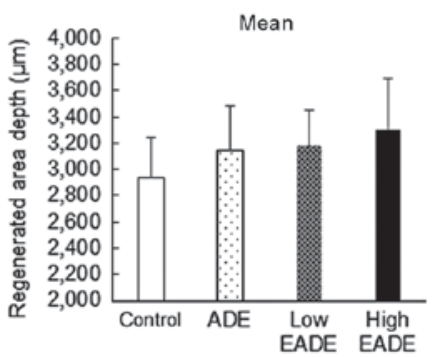

Figure 3. Restoration depth of the cartilage matrix and subchondral matrix based on hematoxylin and eosin staining. The graphs present the depth of the regenerated area in the cartilage matrix for the (A) trochlear sulcus, (B) medial trochlear ridge and (C) overall mean; and the depth of the regenerated area in the subchondral bone matrix for the (D) trochlear sulcus, (E) medial trochlear ridge and (F) overall mean. "P<0.05. ADE, Ajuga decumbens extract; EADA, extra ADE.

A

a

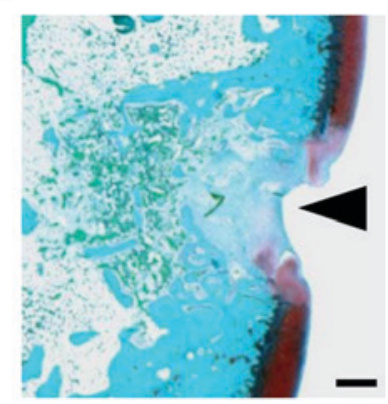

B b

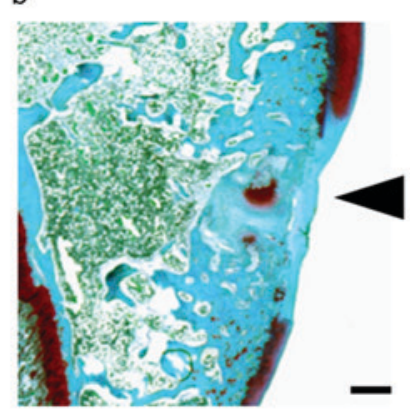

c
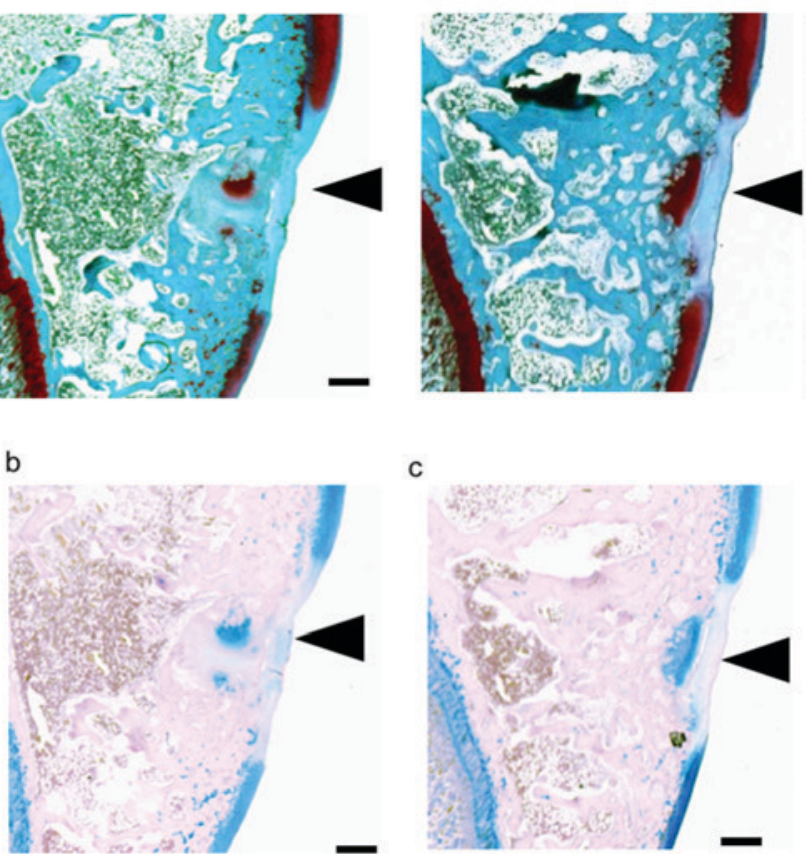

C

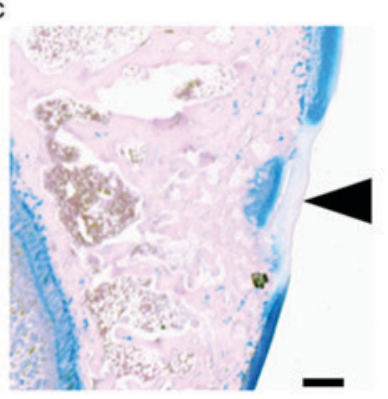

d

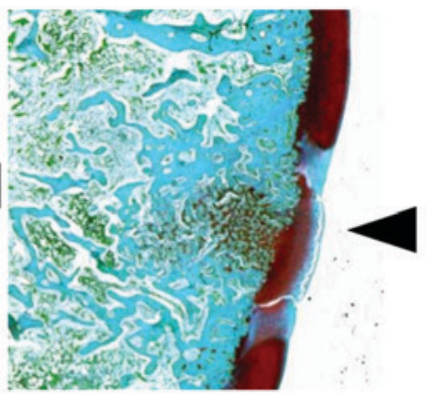

d

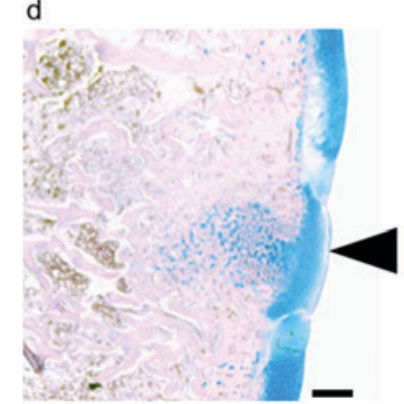

Figure 4. (A) Safranin O and (B) Alcian blue staining in the trochlear sulcus at 3 weeks post-surgery. Proteoglycans in the cartilage matrix were stained with Safranin O (red). Black arrowheads indicate damaged tissue areas. At the trochlear sulcus, red staining was weak in the (Aa) control group, whereas it was stronger in the (Ab) ADE, $(\mathrm{Ac})$ low EADE and (Ad) high EADE groups. The holes were filled with collagen fibers (green) in the control, ADE, low EADE and high EADE groups. The cartilage matrix was stained red to the greatest extent in the high EADE group. The glycosaminoglycans in the cartilage matrix were stained with Alcian blue (blue), which was of similar intensity to the Safranin O staining. Compared with the (Ba) control group, defects were filled with proteoglycan in the $(\mathrm{Bb}) \mathrm{ADE},(\mathrm{Bc})$ low EADE and $(\mathrm{Bd})$ high EADE groups. The cartilage matrix was stained blue in the high EADE group. Scale bar, $500 \mu \mathrm{m}$. ADE, Ajuga decumbens extract; EADA, extra ADE.

were observed between the groups (Fig. 7D-F), though slight decreases were observed in all treatment groups compared with the control excluding for the ADE group at the trochlear sulcus. Collectively these data suggest that EADE activates osteogenesis in subchondral bone.

\section{Effect of EADE in vitro}

Effect of EADE on chondrogenic differentiation in hMSCs. To determine whether chondrogenic differentiation is associated with cartilage matrix regeneration, MSC differentiation to chondrocytes following EADE treatment was assessed. 
A

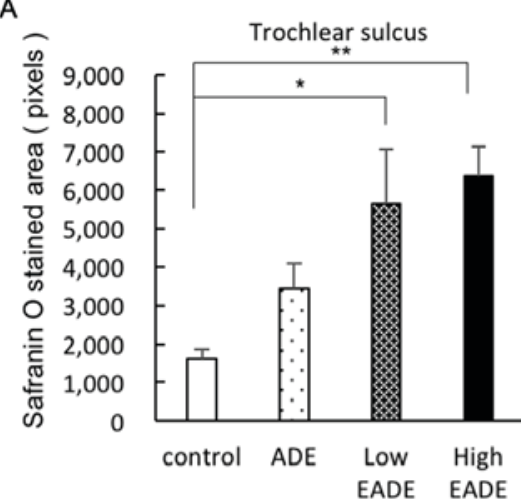

D

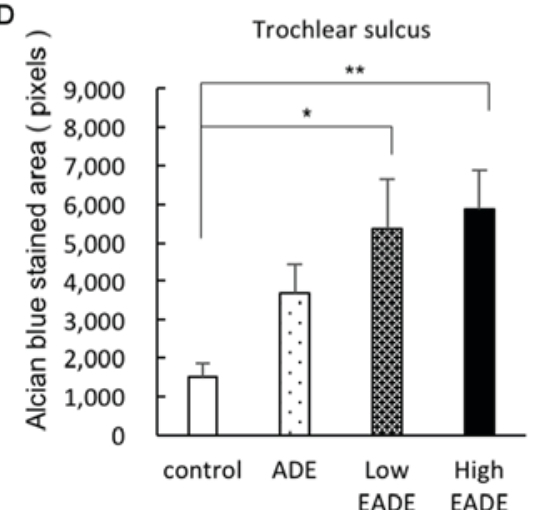

B

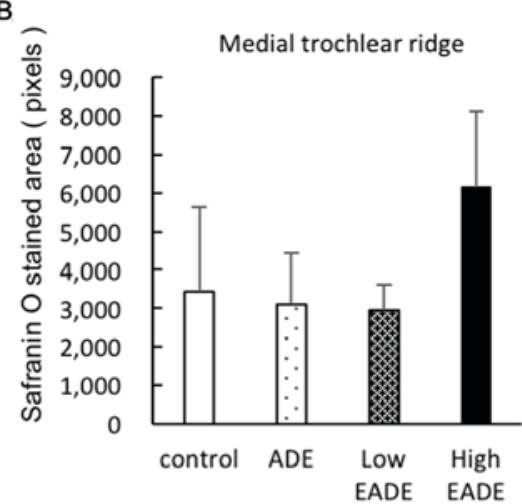

E

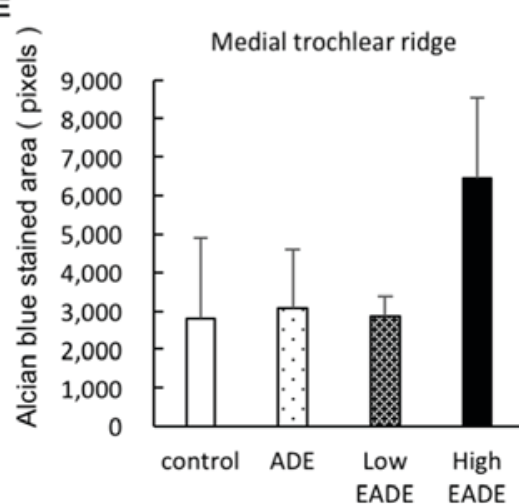

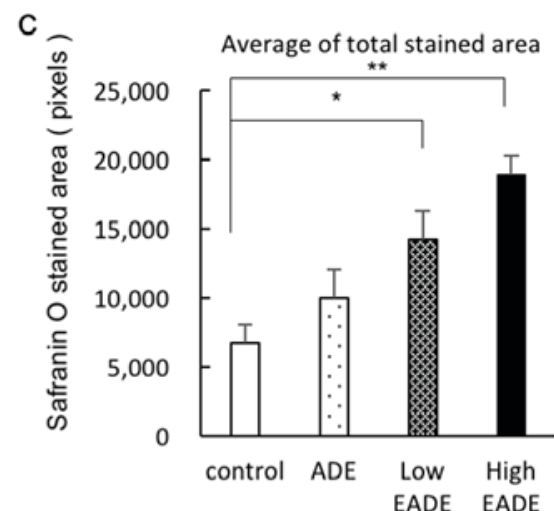

F

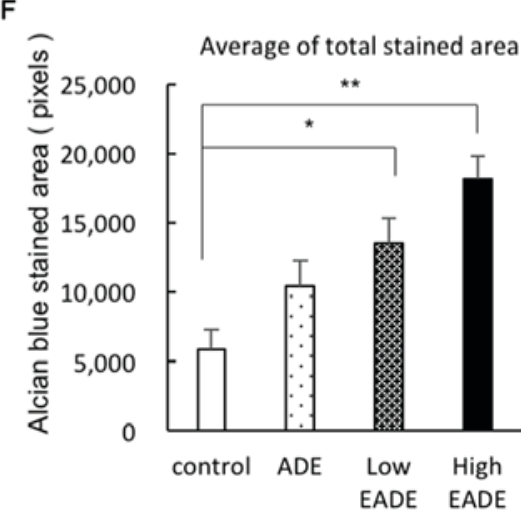

Figure 5. Image analysis of the cartilage matrix following Safranin $\mathrm{O}$ and Alcian blue staining. The graphs present the number of red pixels for the (A) trochlear sulcus, (B) medial trochlear ridge and (C) the overall mean following Safranin O staining; and the number of indigo pixels for the (D) trochlear sulcus, (E) medial trochlear ridge and (F) overall mean following Alcian blue staining. Increases in the stained area indicated enhanced PG and GAG content in the cartilage matrix. ${ }^{*} \mathrm{P}<0.05$ and ${ }^{* *} \mathrm{P}<0.01$. ADE, Ajuga decumbens extract; EADA, extra ADE.
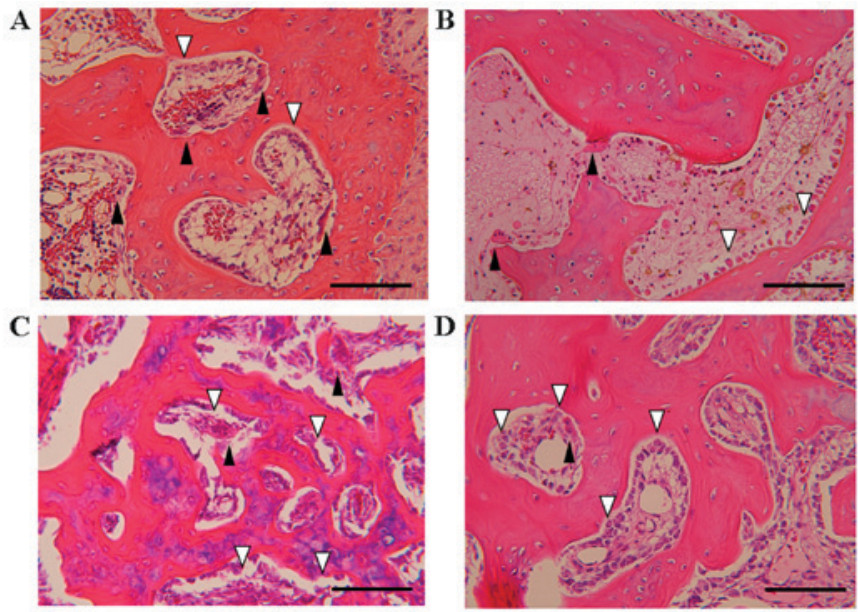

Figure 6. Hematoxylin and eosin staining of subchondral bone at 3 weeks post-surgery. (A) In the control group, there was a marked number of osteoclasts and few osteoblasts. (B) In the ADE group, fewer osteoclasts were observed. In the (C) low and (D) high EADE groups, the number of osteoblasts was markedly increased compared with the control group. Black arrowheads indicate osteoclasts and white arrowheads indicate osteoblasts. Scale bar, $500 \mu \mathrm{m}$.

The results of cytochemical analysis by Alcian blue staining following 3 weeks of hMSC culture are presented in Fig. 8. The hMSCs were cultured for 3 weeks with normal growth medium or chondrogenic differentiation medium with or without EADE $(1-100 \mu \mathrm{g} / \mathrm{ml})$. While undifferentiated MSCs have little extracellular matrix, chondrogenic differentiation results in the formation of cartilage with a typical extracellular matrix composed of PG aggrecan and other glycosaminoglycans (15). PG aggrecan and other glycosaminoglycans are therefore used as indicators of cartilage formation (15), and were thus detected by Alcian blue staining in the present study. The analysis indicated that EADE treatment increased staining associated with aggrecan and other glycosaminoglycans in cells cultured in chondrogenic differentiation medium $(\mathrm{P}<0.05)$ in an apparent dose-dependent manner (Fig. 8).

Effect of EADE on $P G E_{2}$ production in chondrocytes. The repressive effect of EADE on $\mathrm{PGE}_{2}$ production was measured to evaluate its effect on osteogenesis. For control cells cultured without IL-1 $\beta$, a small amount of $\mathrm{PGE}_{2}$ was observed in the cultured cells (Fig. 9). By contrast, cells cultured in the presence of IL-1 $\beta(1 \mathrm{ng} / \mathrm{ml})$ produced PGE $_{2}$ at a markedly higher level compared with the control cells $(\mathrm{P}<0.05)$. The effect of EADE on IL-1 $\beta$-induced $\mathrm{PGE}_{2}$ production was also assessed. Co-culture with 10 or $100 \mu \mathrm{g} / \mathrm{ml}$ EADE significantly blocked the stimulation of $\mathrm{PGE}_{2}$ production by IL-1 $\beta(\mathrm{P}<0.05)$ in an apparent dose-dependent manner (Fig. 9).

\section{Discussion}

In the present study, it was investigated whether the concentrate of the effective fraction of ADE was effective in the treatment of $\mathrm{OA}$ and cartilage regeneration. In a cartilage 
A

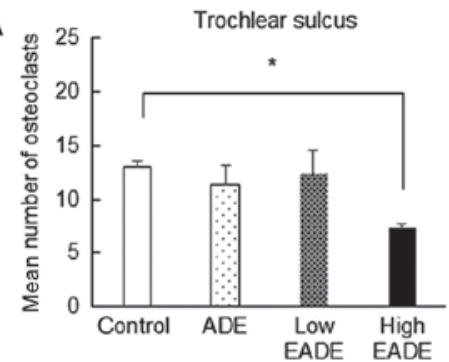

D

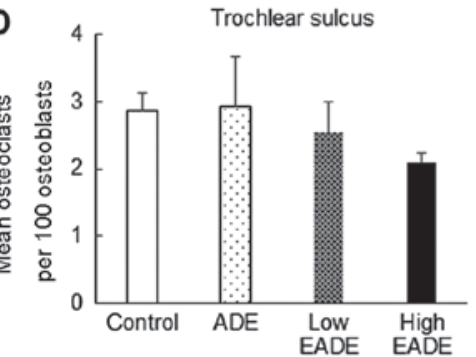

B

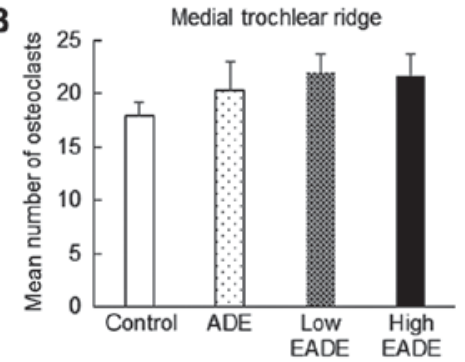

E

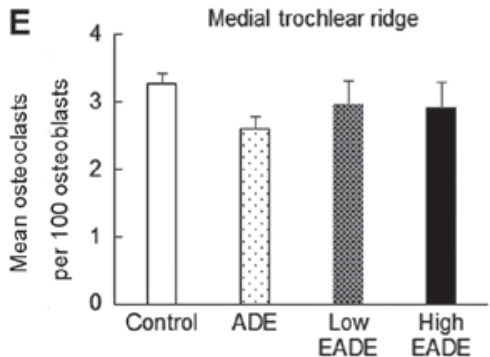

C
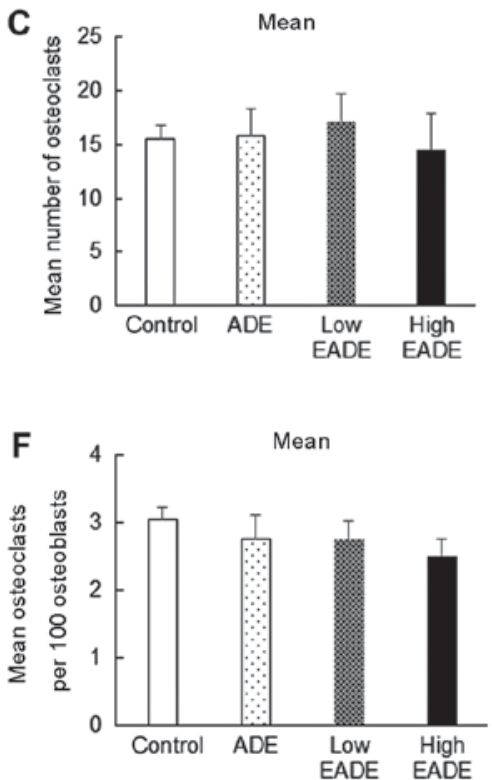

Figure 7. Cell counting of osteoblasts and osteoclasts in the subchondral bone. Mean number of osteoclasts at the surface of the subchondral bone for the (A) trochlear sulcus, (B) medial trochlear ridge and (C) overall mean. Mean number of osteoblasts per 100 osteoblasts in the subchondral bone for the (D) trochlear sulcus, (E) medial trochlear ridge and $(\mathrm{F})$ overall mean. ${ }^{*} \mathrm{P}<0.05$. ADE, Ajuga decumbens extract; EADA, extra ADE.

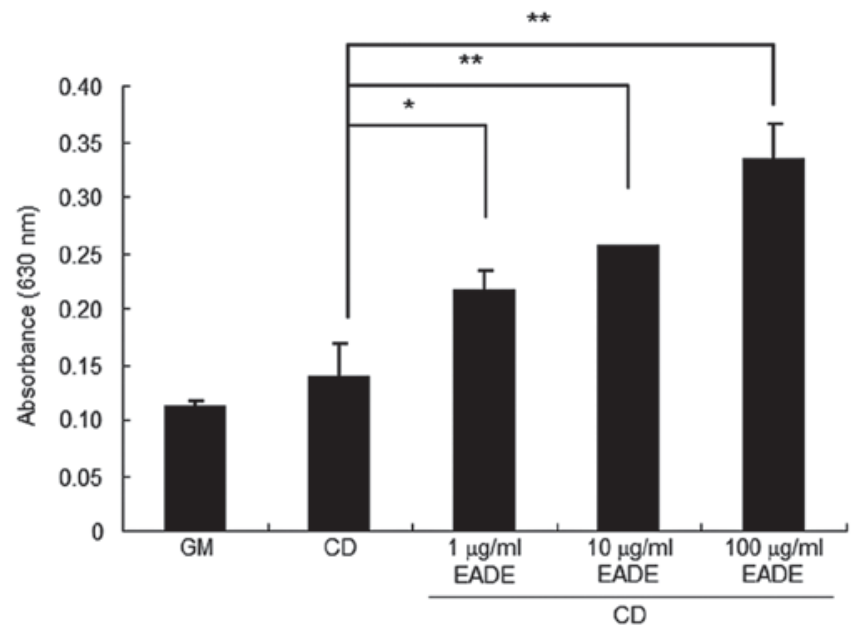

Figure 8. Chondrocyte differentiation from hMSCs. hMSCs were cultured for 3 weeks with GM or CD and different concentrations of EADE. Cells were subjected to Alcian blue staining and the absorbance was measured at $630 \mathrm{~nm}$. ${ }^{*} \mathrm{P}<0.05$ and ${ }^{* *} \mathrm{P}<0.01$. hMSCs, human mesenchymal stem cells; GM, normal growth medium; $\mathrm{CD}$, chondrogenic differentiation medium; EADE, extra Ajuga decumbens extract.

injury model, the degree of restoration within surgical holes was increased in an apparent dose-dependent manner by EADE. In particular, the cartilage matrix was significantly regenerated in the EADE groups compared with the control group and the regeneration area was improved in the EADE treatment groups, most notably in the high EADE group, as demonstrated by Safranin O and Alcian blue staining. The data obtained from Safranin O and Alcian blue staining were consistent and the extent of staining exhibited an apparent correlation with the concentration of EADE, which suggests that this compound increases the amount of GAGs and PGs in cartilage matrix. In a previous study by our group, the number of osteoclasts was significantly decreased following

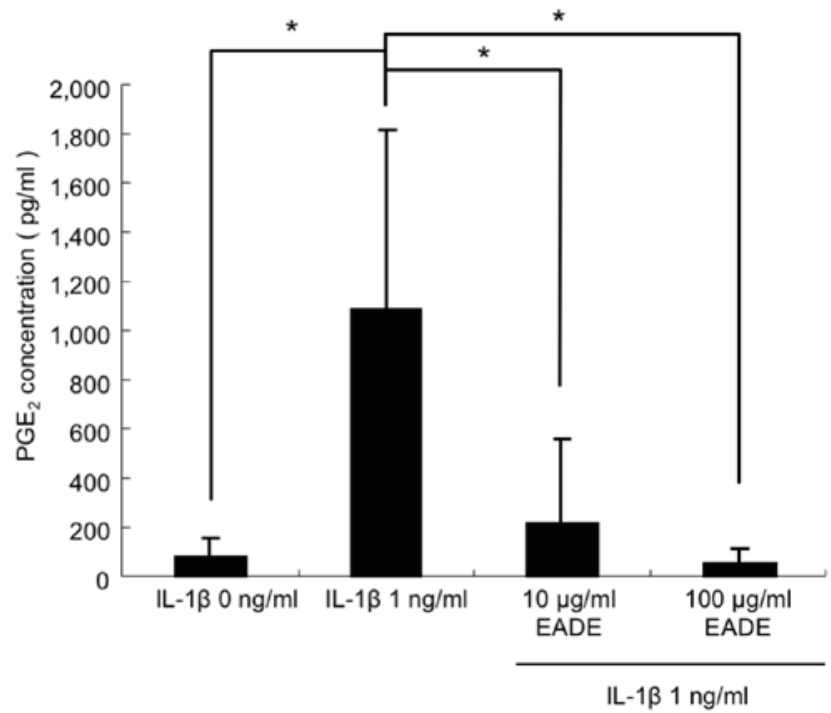

Figure 9. Effect of EADE on PGE2 production in chondrocytes. Human chondrocytes were stimulated with IL-1 $\beta(1 \mathrm{ng} / \mathrm{ml})$ and treated with 10 or $100 \mu \mathrm{g} / \mathrm{ml}$ EADE. The concentration of PGE2 was significantly increased by IL-1 $\beta$ stimulation, and EADE significantly suppressed this increase. ${ }^{*} \mathrm{P}<0.05$. IL, interleukin; EADE, extra Ajuga decumbens extract; PGE2, prostaglandin E2.

administration of ADE or 20-hydroxyecdysone (11); however, its influence on bone metabolism was unclear. Bone metabolism is regulated by a balance between the functions of osteoclast and osteoblast cells (17). ADE has previously been demonstrated to inhibit osteoclast differentiation (10) and 20-hydroxyecdysone has also been reported to have beneficial effects in epiphyseal cartilage and trabecular bone in ovariectomized rats (12). In the present study, the mean number of osteoclasts per 100 osteoblasts in the subchondral bone was decreased to the greatest extent in the high ADE group compared with the control group. These results suggest 
that EADE may influence the number of osteoclasts as well as bone metabolism and the regeneration of subchondral bone. It has been reported that 20-hydroxyecdysone stimulates MSC osteogenic differentiation (18), and so as an active component in EADE, it may stimulate MSCs and enhance osteogenesis in subchondral bone.

When cartilage is injured, natural healing is slow and typically results in the formation of nonfunctional fibro-cartilage, while regeneration of hyaline cartilage rarely occurs (7). However, the administration of GlcN can regenerate hyaline cartilage (7). Experimental data have demonstrated that the mechanism underlying this action is associated with chondroblast activation (19). Glucosamine promotes a chondrogenic phenotype in MSCs (20) and inhibits matrix metalloproteinase (MMP)-13 expression and matrix degradation (20). MMPs and IL-1 $\beta$ have been demonstrated to serve a role in the degradation of articular cartilage (21-23). IL-1 $\beta$ is produced by mononuclear cells in the arthritic synovium and chondrocytes (21) and enhances the production of various chemical mediators, including $\mathrm{PGE}_{2}(22)$, nitric oxide (NO) and MMPs (23), in chondrocytes. In the present study, the ability of EADE to induce MSC differentiation and its anti-inflammatory effects in chondrocytes were assessed in order to investigate the mechanisms underlying the regeneration process following cartilage injury. EADE was indicated to stimulate $\mathrm{PG}$ production and induce in vitro chondrogenic differentiation of MSCs. Furthermore, EADE (10-100 $\mu \mathrm{g} / \mathrm{ml})$ significantly attenuated IL-1 $\beta$-induced $\mathrm{PGE}_{2}$ production in chondrocytes. It has been reported that 20-hydroxyecdysone suppresses IL-1 $\beta$-induced catabolic gene expression in cartilage (24) and that ADE inhibits the expression of iNOS and NO production in macrophages (10). It has also been documented that $\mathrm{PGE}_{2}$ may induce receptor activator of nuclear factor kappa-B ligand (RANKL) expression in osteoblasts and directly enhance RANKL-induced osteoclastogenesis in precursors (25). In addition, 20-hydroxyecdysone has been demonstrated to stimulate MSC osteogenic differentiation (18), suggesting that 20-hydroxyecdysone may suppress IL-1 $\beta$-induced inflammation and $\mathrm{PGE}_{2}$-induced RANKL expression. EADE may therefore have an anti-inflammatory effect and suppress osteoclastogenesis.

In conclusion, the present study investigated the effect of EADE on the acceleration of healing in an experimental model of cartilage injury. The cartilage matrix, along with the subchondral matrix, was markedly regenerated in the low and high EADE groups. Additionally, EADE stimulated PG production, induced in vitro chondrogenic differentiation of MSCs, and significantly attenuated IL-1 $\beta$-induced $\mathrm{PGE}_{2}$ production in chondrocytes. Thus, EADE not only exerted a chondroprotective effect, but also influenced bone metabolism and stimulated subchondral bone restoration. The results of the present study suggest that EADE may be an effective treatment for cartilaginous damage and have greater therapeutic effect than the currently used therapeutic ADE. The present study only investigated the effect of EADE on improving knee destruction in vivo and in vitro. Further study is required to determine whether EADE may improve the join function and QOL in patients with knee injuries.

\section{Acknowledgements}

The authors would like to thank Professor Saburo Minami (Tottori University, Tottori, Japan) for his valuable advice and Mr. Shohei Hishikawa (Tottori University) for his assistance.

\section{References}

1. Yoshimura N, Muraki S, Oka H, Mabuchi A, En-Yo Y, Yoshida M, Saika A, Yoshida H, Suzuki T, Yamamoto S, et al: Prevalence of knee osteoarthritis, lumbar spondylosis, and osteoporosis in Japanese men and women: The research on osteoarthritis/osteoporosis against disability study. J Bone Miner Metab 27: 620-628, 2009 .

2. Buckwalter JA, Martin J and Mankin HJ: Synovial joint degeneration and the syndrome of osteoarthritis. Instr Course Lect 49: 481-489, 2000.

3. Buckwalter JA, Roughley PJ and Rosenberg LC: Age-related changes in cartilage proteoglycans: Quantitative electron microscopic studies. Microsc Res Tech 28: 398-408, 1994.

4. Martin JA and Buckwalter JA: Roles of articular cartilage aging and chondrocyte senescence in the pathogenesis of osteoarthritis. Iowa Orthop J 21: 1-7, 2001.

5. Reginster JY, Deroisy R, Rovati LC, Lee RL, Lejeune E, Bruyere O, Giacovelli G, Henrotin Y, Dacre JE and Gossett C: Long-term effects of glucosamine sulphate on osteoarthritis progression: A randomised, placebo-controlled clinical trial. Lancet 357: 251-256, 2001.

6. Nakamura H, Shibakawa A, Tanaka M, Kato T and Nishioka K: Effects of glucosamine hydrochloride on the production of prostaglandin E2, nitric oxide and metalloproteases by chondrocytes and synoviocytes in osteoarthritis. Clin Exp Rheumatol 22: 293-299, 2004.

7. Tamai Y, Miyatake K, Okamoto Y, Takamori Y, Sakamoto H and Minami S: Enhanced healing of cartilaginous injuries by glucosamine hydrochloride. Carbohydr Polym 48: 369-378, 2002.

8. Naito K, Watari T, Furuhata A, Yomogida S, Sakamoto K, Kurosawa H, Kaneko K and Nagaoka I: Evaluation of the effect of glucosamine on an experimental rat osteoarthritis model. Life Sci 86: 538-543, 2010.

9. Clegg DO, Reda DJ, Harris CL, Klein MA, O'Dell JR, Hooper MM, Bradley JD, Bingham CO III, Weisman MH, Jackson CG, et al: Glucosamine, chondroitin sulfate, and the two in combination for painful knee osteoarthritis. N Engl J Med 354: 795-808, 2006.

10. Ono Y, Fukaya Y, Imai S and Yamakuni T: Beneficial effects of Ajuga decumbens on osteoporosis and arthritis. Biol Pharm Bull 31: 1199-1204, 2008.

11. Sawada Y, Sugimoto A, Fukuda K, Kurosawa T, Ogawa M, Osaki T and Minami S: Oral administration of Ajuga decumbens extract has a synergetic effect with glucomsaine on cartilaginous injury in a rabbit osteoaruthritis model. J Chitin Chitosan Sci 2: 191-196, 2014.

12. Kapur P, Wuttke W, Jarry H and Seidlova-Wuttke D: Beneficial effects of beta-Ecdysone on the joint, epiphyseal cartilage tissue and trabecular bone in ovariectomized rats. Phytomedicine 17: 350-355, 2010.

13. Mardones R, Jofré CM and Minguell JJ: Cell therapy and tissue engineering approaches for cartilage repair and/or regeneration. Int J Stem Cells 8: 48-53, 2015.

14. Ham O, Lee CY, Kim R, Lee J, Oh S, Lee MY, Kim J, Hwang KC, Maeng LS and Chang W: Therapeutic potential of differentiated mesenchymal stem cells for treatment of osteoarthritis. Int J Mol Sci 16: 14961-14978, 2015.

15. Johnson K, Zhu S, Tremblay MS, Payette JN, Wang J, Bouchez LC, Meeusen S, Althage A, Cho CY, Wu X and Schultz PG: A stem cell-based approach to cartilage repair. Science 336: 717-721, 2012.

16. Osaki T, Kitahara K, Okamoto Y, Imagawa T, Tsuka T, Miki Y, Kawamoto H, Saimoto H and Minami S: Effect of fucoidan extracted from mozuku on experimental cartilaginous tissue injury. Mar Drugs 10: 2560-2570, 2012.

17. Rodan G: Introduction to bone biology. Bone 13 (Suppl 1) S3-S6, 1992.

18. Gao L, Cai G and Shi X: Beta-ecdysterone induces osteogenic differentiation in mouse mesenchymal stem cells and relieves osteoporosis. Biol Pharm Bull 31: 2245-2249, 2008. 
19. Hashida M, Miyatake K, Okamoto Y, Fujita K, Matsumoto T, Morimatsu F, Sakamoto K and Minami S: Synergistic effects of D-glucosamine and collagen peptides on healing experimental cartilage injury. Macromol Biosci 3: 596-603, 2003.

20. Derfoul A, Miyoshi AD, Freeman DE and Tuan RS: Glucosamine promotes chondrogenic phenotype in both chondrocytes and mesenchymal stem cells and inhibits MMP-13 expression and matrix degradation. Osteoarthritis Cartilage 15: 646-655, 2007.

21. Farahat MN, Yanni G, Poston R and Panayi GS: Cytokine expression in synovial membranes of patients with rheumatoid arthritis and osteoarthritis. Ann Rheum Dis 52: 870-875, 1993.

22. Campbell IK, Piccoli DS and Hamilton JA: Stimulation of human chondrocyte prostaglandin E2 production by recombinant human interleukin-1 and tumour necrosis factor. Biochim Biophys Acta 1051: 310-318, 1990.
23. Tetlow LC, Adlam DJ and Woolley DE: Matrix metalloproteinase and proinflammatory cytokine production by chondrocytes of human osteoarthritic cartilage: Associations with degenerative changes. Arthritis Rheum 44: 585-594, 2001.

24. Sheu SY, Ho SR, Sun JS, Chen CY and Ke CJ: Arthropod steroid hormone (20-Hydroxyecdysone) suppresses IL-1 $\beta$-induced catabolic gene expression in cartilage. BMC Complement Altern Med 15: 1, 2015.

25. Kotake S, Yago T, Kawamoto M and Nanke Y: Effects of NSAIDs on differentiation and function of human and murine osteoclasts-crucial 'human osteoclastology'. Pharmaceuticals (Basel) 3: 1394-1410, 2010.

(D) $\Theta$ This work is licensed under a Creative Commons Attribution-NonCommercial-NoDerivatives 4.0 International (CC BY-NC-ND 4.0) License. 